\title{
Automatic Gait Generation in Modular Robots: "to Oscillate or to Rotate; that is the question"
}

\author{
Soha Pouya, Jesse van den Kieboom, Alexander Spröwitz, Auke Jan Ijspeert
}

\begin{abstract}
Modular robots offer the possibility to design robots with a high diversity of shapes and functionalities. This nice feature also brings an important challenge: namely how to design efficient locomotion gaits for arbitrary robot structures with many degrees of freedom.

In this paper, we present a framework that allows one to explore and identify highly different gaits for a given arbitraryshaped modular robot. We use simulated robots made of several Roombots modules that have three rotational joints each. These modules have the interesting feature that they can produce both oscillatory movements (i.e. periodic movements around a rest position) and rotational movements (i.e. with continuously increasing angle), leading to very rich locomotion patterns. Here we ask ourselves which types of movements - purely oscillatory, purely rotational, or a combination of both- lead to the fastest gaits. To address this question we designed a control architecture based on a distributed system of coupled phase oscillators that can produce synchronized rotations and oscillations in many degrees of freedom. We also designed a specific optimization algorithm that can automatically design hybrid controllers, i.e. controllers that use oscillations in some joints and rotations in others, for fast gaits. The proposed framework is verified by multiple simulations for several robot morphologies. The results show that (i) the question whether it is better to oscillate or to rotate depends on the morphology of the robot, and that in general it is best to do both, (ii) the optimization framework can successfully generate hybrid controllers that outperform purely oscillatory and purely rotational ones, and (iii) the resulting gaits are fast, innovative, and would have been hard to design by hand.
\end{abstract}

\section{INTRODUCTION}

Modular robots present an interesting platform to explore locomotion strategies for robotics. Indeed, their (self-) reconfigurability allows one to explore various types of gaits in multiple types of morphologies. However, the large variety in robot configurations and having many degrees of freedom make it problematic for the user to imagine all different solutions. Hand coding and editing the gaits is tiring and timeconsuming. Moreover, there might always be some solutions which are not explored by the designer. Therefore design tools are needed to help extract the capabilities of a newly designed modular robot. Here, our goal is two-fold: (i) to present a framework for automatically designing locomotion controllers for arbitrary robot morphologies, and (ii) to use that framework to explore whether oscillations, rotations, or combinations of both, lead to the fastest locomotion. Some impressive locomotor performance can be obtained by either type of movement (see for instance the Big Dog robot for locomotion based on oscillatory movements [1] and

Authors are with Biorobotics Laboratory, Institute of Bioengineering, School of engineering, Ecole Polytechnique Fédérale de Lausanne (EPFL), first-name.last-namedepfl.ch
Rhex and Whegs robots for rotational movements [2]), but combinations of both are rarely used (see the salamander robot in [3] for an exception). Furthermore, the questions of which type of movements are best for a given morphology, and whether combining them could lead to even better performance, have not yet been addressed to the best of our knowledge.

The framework that we propose has two components: a distributed locomotion controller and an optimization algorithm that performs both structural and parametric optimization. The locomotion controller is implemented in a distributed system of coupled oscillators one per degree of freedom similar to the concept of central pattern generators (CPGs) found in the spinal cord of vertebrate animals. The CPGs are based on coupled phase oscillators to ensure synchronized behavior and have different output filters to allow switching between oscillations and rotations. The optimization algorithm is a modified Particle Swarm Optimization (PSO) algorithm that optimizes both the structure of the controller (i.e. which type of movement is used for each degree of freedom) and its parameters (for instance amplitude and phase difference between oscillators).

\section{A. Related Work}

Most projects in modular robotics have used oscillations for generating forward locomotion [4]-[7]. Rotational movements have shown interesting gaits using wheeled or Whegslike [8] propulsion. Combining these two modes sounds like an interesting approach to derive newer gaits. Similar to this idea, Hancher et al. [5] used rotation in wheeled-shape modules and oscillation in different type of modules for the rest of the robot shape. Zykov et al. [9] have recently extended Molecubes with several active and passive modules to diversify robot capabilities. In related work, we designed a salamander robot that uses rotational movements for the limbs and oscillatory movements for the spine [3]. However, it has not been explored how a combination of different movements in all the joints can influence the resulting gaits. In particular, we are interested in comparing the functionality of oscillatory and rotational movements and also their combinations in hybrid solutions. The Roombots modules presented below allow to investigate these questions because of their capability to perform both oscillation and continuous rotation. Different locomotion control algorithms have been developed for modular robots. As one of the pioneers in the field, Yim [10] proposed gait table control which uses sequences of time-driven actions for modules. In a recent development, Zhang et al [11] use a new form of gait 
tables called Phase Automata which uses event-driven state machines. These methods usually use predefined sequences but can be adjusted during runtime [12]. Role-based control [13], hormone-based control [14] and constraint-based control [15] have been used for locomotion control with more concentration on periodic movements for the joints. Furthermore, several works have suggested using central pattern generators (CPGs) as the locomotion control [16] [6]. CPGs are networks of coupled nonlinear oscillators capable of producing robust, synchronized movement patterns with minimum number of control parameters which makes them well-suited for optimization algorithms. Similar to the seminal work of Sims [17], Zykov et al. [4] used a genetic algorithm (GA) to evolve the controller parameters with rhythmic locomotion patterns. Sproewitz [6] used Powell's method as the online learning procedure for evolving the CPG parameters. Hancher and Hornby [5] used steady-state evolutionary algorithm while assuming fixed morphology and a periodic gait. Christensen et al. [7] use a machine learning approach to evolve the parameters of the controller which can generate different actions of rotation and stopping.

In this paper, a CPG is used to produce synchronized patterns for oscillatory and rotational joint movements actuating a Roombots structure. A modified version of particle swarm optimization (PSO) provides the optimization of both the CPG structure and parameters. The remainder of the paper is organized as follows. We first describe the Roombots hardware in section II. Section III then presents the applied controller architecture including the CPG model and topology. In section IV the proposed algorithm for optimal gait generation is presented. Sections V and VI describe the experiments and their results. The article is concluded with a discussion and an outlook on future work in section VII.

\section{ROOMBOTS HARDWARE}

Roombots modular robots [18] are fitted into a regular cubic grid with $110 \mathrm{~mm}$ edge length. We connect two Roombots modules serially into a $R B$ metamodule. Any of the three joints of a RB unit delivers sufficient torque to rotate a metamodule in the "worst case scenario situation", i.e. out of a horizontal stretched position (see Table I for property details). Roombots shell elements are printed in ABS plastics, plate-elements are milled out of FR4 material. A RB module weights about $1.4 \mathrm{~kg}$, that includes batteries and the estimated weight for electronic boards ${ }^{1}$. We use a relatively high gear ratio (around $380: 1$ ) to achieve necessary torques, what limits maximum rotational speed. The center RB joint needs $3 \mathrm{sec}$ to rotate $360^{\circ}$, both outer joints roughly $2 \mathrm{sec}$.

The active connection mechanism is genderless, four-way symmetric, has four mechanical latching fingers [19], and its fingers are completely retractable. It is roughly $65 \mathrm{~mm}$ in diameter, and $19 \mathrm{~mm}$ in height. In many ways the connector design is similar to the AMAS connection mechanism [20], although we use a different trajectory for the movement of the latching fingers. Initial connector tests indicate a passive

\footnotetext{
${ }^{1}$ Roombots' electronics are under construction
}

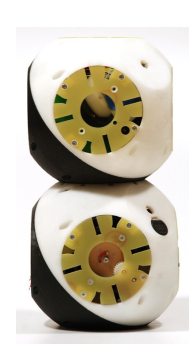

(a) One RB unit.

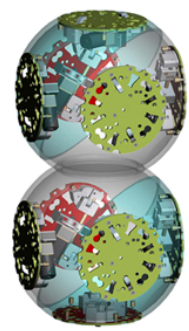

(b) ACMs.

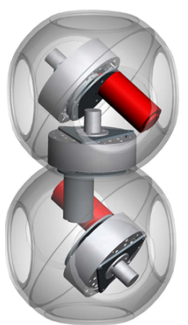

(c) Actuators.

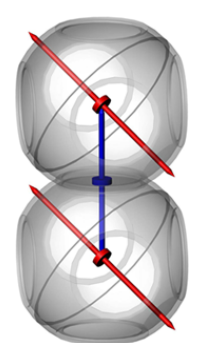

(d) $3 \mathrm{DOF}$
Fig. 1: (a) One Roombots module. (b) Four active connection mechanisms are mounted into one RB module. (c) A custom designed motor-gearbox combination. (d) All RB axes are continuous rotational.

TABLE I: Roombots module hardware specifications

\begin{tabular}{lll}
\hline Type & Details & $\#$ \\
\hline \hline Main joints & continuous rotational & 3 \\
Size & $110 \mathrm{~mm} \times 110 \mathrm{~mm} \times 220 \mathrm{~mm}$ & \\
Weight & $1.4 \mathrm{~kg}$ & 2 \\
$M_{O}$ & $7 \mathrm{Nm}$ & 1 \\
$M_{i}$ & $5 \mathrm{Nm}$ & 2 \\
Outer gears & $370: 1$, spur and planetary & 1 \\
Inner gears & $380: 1$, spur and planetary & 4 \\
ACM & 4 way symmetric, genderless, retractable & 1 \\
ACM actuator & mini DC motor & 3 \\
Others & 12-line slip rings & \\
\hline
\end{tabular}

tolerance against alignment errors of roughly $2 \mathrm{~mm}$ between modules. To built a metamodule a RB units' foot hemisphere can be attached to the head hemisphere of the second RB unit in one of the following modes, where each mode refers to the relative orientation of the hemispheres axes: parallel (PAR), perpendicular (PER), shear-S (SRS), and shear-Z (SRZ) (see also figure $4 a)$.

\section{Controller Architecture}

This section describes the CPG locomotion controller. Control inputs for the CPG are high level parameters such as amplitude, offset and phase lags. Each oscillator is capable of producing either oscillatory or rotational joint angle signals. ${ }^{2}$ We apply an oscillator network topology which matches the hardware topology, e.g. a quadruped structure or a single Roombots metamodule. An evolutionary algorithm provides an automatic design of the control input parameters.

\section{A. CPG Model}

We designed a CPG controller which can produce two types of basic movements for each DOF: (i) Rotational movements that result from a continuously rotating (swivel) joint, and can provide wheel or Whegs-like [8] propulsion, and (ii) Oscillatory movements that periodically oscillate around a resting position. Since it is important for stable, reproducible locomotion to keep all DOF synchronized whatever their mode, we built the controller as a distributed

\footnotetext{
${ }^{2}$ In the remaining part of the paper we will use oscillator to refer to pattern generators capable of producing both oscillatory and rotational output.
} 
system of coupled phase oscillators, with one oscillator per DOF (joint) $i$ :

$$
\begin{aligned}
& \dot{\phi}_{i}=2 \pi \cdot \omega_{i}+K_{i}+f_{\theta_{i}}(\vec{s}) \\
& K_{i}=\sum_{j} w_{i j} \cdot r_{j} \cdot \sin \left(\phi_{j}-\phi_{i}-\psi_{i j}\right) \\
&\left.\left.\begin{array}{rl}
\dot{r}_{i}= & a_{i}\left(R_{i}-r_{i}\right)+f_{r_{i}}(\vec{s}) \\
\theta_{i}=r_{i} \cdot \sin \left(\phi_{i}\right)+X_{i} & (\text { Oscillation }) \\
\theta_{i}= & \phi_{i} \\
\theta_{i}=X_{i} & (\text { Rotation })
\end{array}\right\} \text { Lervo inputs }\right)
\end{aligned}
$$

where $\theta_{i}$ is the servo input which can be derived with different functions corresponding to the desired servo movement. Variables $r_{i}$ and $\phi_{i}$ are state variables which encode amplitude and phase of the oscillation. The parameters $w_{i j}$ and $\psi_{i j}$ are respectively the coupling weight and phase bias of the coupling between oscillators $i$ and $j . a_{i}$ is a positive constant which determines the rise time of the amplitude to the desired value $R_{i}$. The parameters $R_{i}, X_{i}$, and $\psi_{i j}$ are open parameters of which a subset (depending on the selected mode) is subject to optimization. Furthermore, this structure is capable of including sensory feedback. ${ }^{3}$ For this purpose the state variables can be influenced by sensory feedback signals through the functions $f_{\theta_{i}}$ and $f_{r_{i}}, \vec{s}$ being a vector of sensor states.

Equation 3 shows three possible modes which result in oscillations, rotations or a locked condition. In the oscillation mode, the output exhibits limit cycle behavior, thus producing a stable periodic trajectory. For rotation a constant-speed profile is generated leading to a monotonic increase of the joint angle. We also include a third mode which allows the controller to lock a joint.

With the right parameter values, rotational and oscillatory DOF will rapidly converge to a phase-locked regime, i.e. a regime with a constant phase difference even between phase oscillators that are in different modes. This is highly desirable for the implementation of stable, coordinated gaits. It will also ensure that several joints remain phased-locked, even if they are controlled by oscillators implemented on different micro-controllers with slightly different clocks. Fig. 2 shows this synchronization behavior between three DOFs, with two activated in oscillation mode and one in rotation mode.

\section{B. CPG Topology}

When designing CPGs, the network coupling parameters $w_{i j}$ and $\phi_{i j}$ between different oscillators are of importance. For known types of locomotion gait patterns, such as quadrupedal or snake gaits, the coupling architecture can be specified based on biological observations. Here the goal is to find different and unexpected gaits, which an arbitrarily shaped modular robot could potentially create. Hence we do not specify a pre-defined oscillator network topology. We let the coupling structure of the CPG correspond the robot's morphology, i.e. phase oscillators of neighbor DOF are coupled together. We use one common frequency for

\footnotetext{
${ }^{3}$ Note that sensory feedback is not applied in this article.
}
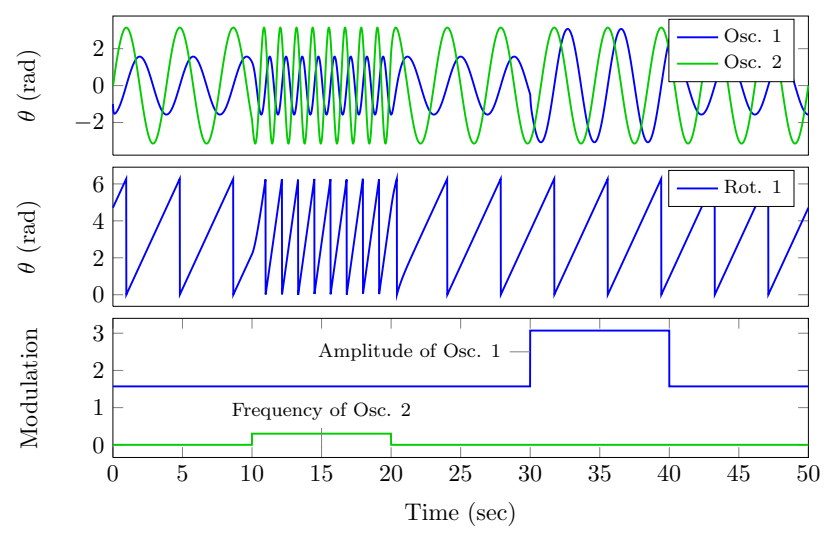

Fig. 2: Synchronization behavior of three coupled oscillators: two in oscillatory mode (upper plot) and one in rotational mode (middle plot). All oscillators are coupled, hence synchronize within the first seconds. Frequency modulation from $t=10$ to $20 \mathrm{sec}$, amplitude modulation from $t=30$ to $40 \mathrm{sec}$ of the simulation.

all oscillators $(f=0.26 \mathrm{~Hz}$, derived from the hardware implementation) and symmetric bi-directional couplings. All coupling weights are set to 2 , phase differences $\psi_{i j}$ are open parameters and subject to optimization. We do not induce symmetry artificially, i.e. we do not apply any mirroring of parameter sets along our network. Applying symmetry is usually a good strategy to reduce the number of open parameters. However it also might limit the resulting gaits, as it restricts the possible variety of parameters.

\section{Optimal Gait Generation}

The optimization algorithm can be described by two layers. The outer layer performs structural optimization, and is discrete. The inner layer performs parametric optimization on continuous valued parameters corresponding to the selected movement types.

In this paper, an extension of Particle Swarm Optimization [21] is used to perform the optimization process. PSO is a stochastic, population based optimization method using principles of collaboration rather than competition to evolve individuals. In PSO, each individual is represented by a position and velocity vector, representing respectively the particle's parameter values and search direction. The evolution of each particle in the swarm is then governed by Eq. (4) as suggested in [22].

$$
\begin{aligned}
\overrightarrow{v_{i}}(t+1)= & K \cdot\left[\overrightarrow{v_{i}}(t)+c_{1} r_{1}\left(\overrightarrow{p_{i}}-\overrightarrow{x_{i}}(t)\right)+\right. \\
& \left.c_{2} r_{2}\left(\overrightarrow{p_{g}}-\overrightarrow{x_{i}}(t)\right)\right] \\
\overrightarrow{x_{i}}(t)= & \overrightarrow{x_{i}}(t-1)+\overrightarrow{v_{i}}(t)
\end{aligned}
$$

Where $\overrightarrow{v_{i}}(t)$ is the velocity vector, $\vec{x}(t)$ is the position vector, $K$ is a constriction factor, $c_{1}$ and $c_{2}$ are respectively the cognitive and social factors, $r_{1}$ and $r_{2}$ are two pseudorandom numbers in the range [0 1], $p_{i}$ is the best known solution vector of particle $i$ and $p_{g}$ is the global best known solution vector. The constriction factor, cognitive factor and social factor were set to ensure convergence (see for more detail, [22]). 
The PSO algorithm described thus far is used for the inner layer optimization of the continuous parameters of a specific selection of movement types. Particles are initially uniformly distributed over the possible combinations of movement types. In each such combination, particles share the same parameters, and an independent PSO optimizes their respective solutions. The task of the outer layer is then to do the structural optimization and to move particles from one combination of movement types to another.

The outer layer consists of a set of mutation operators inspired by Genetic Algorithms. Similar to the velocity update of the PSO, the probability of mutation of each actuated degree of freedom is composed of:

- $P_{e}$ : exploration probability of mutation to a movement type (oscillation, rotation or locked) other than the current one

- $P_{c}$ : cognitive probability of mutation to the movement type which is part of the selection with the best results in the particle's history

- $P_{s}$ : social probability of mutation similar to $P_{c}$ but from the best results taken over all the particles

Governed by these three probabilities, particles will be mutated to different combinations of movement types during the optimization process. Once a particle moves to a different parameter space it is incorporated in the PSO running locally in that space.

A main challenge is to choose appropriate values for the different probabilities $P_{e}, P_{c}$ and $P_{s}$. In general, we want to stimulate exploration in the early phases of the optimization, visiting many possible combinations of movement types. Then, as the optimization progresses, particles should start exploring their local known best solutions in more detail. Finally we want the particles to converge in the best known space, as if selecting the best configuration of movement types. The system then starts behaving as a standard PSO with a fixed configuration of movement types as more and more particles are attracted.

The desired behavior can be designed by varying the probabilities $P_{e}, P_{c}$ and $P_{s}$ as the optimization progresses. In this paper, the exploration and social probability were defined using a sigmoid function. The cognitive probability was defined using a gaussian function. Fig. 3 shows the probability characteristics used in all the experiments.

\section{EXPERIMENTAL SETUP}

We performed several experiments applying our CPG- and optimization framework. Firstly we were interested in exploring the locomotion abilities of the four types of metamodules (PAR, PER, SRS, and SRZ), and of two quadruped robot (Fig. 4). Our motivation for testing metamodules is that they represent the simplest possible robot shape built from two Roombots modules (six DOF). In addition, two quadruped shapes were carefully designed featuring symmetry and DOFs allowing quadruped locomotion. These structures were used to verify our approach on more complex shapes with well designed features. Finally, a more arbitrary, asymmetric shape was constructed with no specific design features in

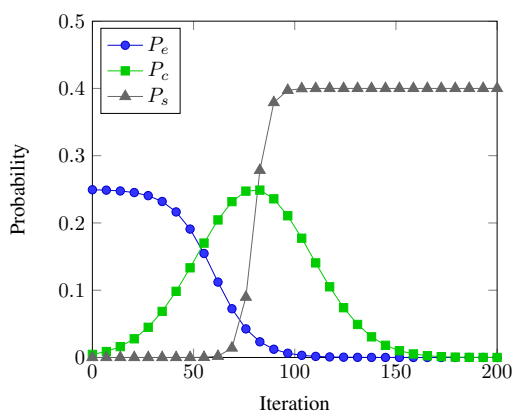

Fig. 3: Mutation probability characteristics for the exploration probability $P_{e}$, cognitive probability $P_{c}$ and social probability $P_{s}$, emphasizing early exploration and late convergence.

mind (Fig. 4c). This allows us to compare the performance of the optimization algorithm on shapes with no pre-designed characteristics, for which a well performing gait can be hard to imagine or design.

We are also interested to know which type of movement, oscillatory or rotational, would lead to the highest locomotion speed. We conducted the following optimization experiments with different possible combinations of the four joint modes: (1) pure rotation, all DOF are in the rotation mode, (2) hybrid rotation with DOF either in rotation or locked mode, (3) pure oscillation, all DOF are in oscillation mode, and (4) fully hybrid, DOF are in oscillation, rotation, or locked mode, and different modes coexist within the robot.

The evolutionary algorithm evolves new solutions, combining structural and parametric optimization. The generated solutions are then evaluated in Webots [23], a simulation tool based on ODE providing simulation of collisions, rigid body dynamics and actuator properties. The average locomotion speed determines the fitness of a particular solution, which is sent back to the optimizer. A RB module/module collision detection penalizes unrealistic solutions with a zero fitness.

\section{RESUlTs AND Discussion}

a) Fitness behaviour and automatic mode selection: Fig. 6 shows the average and standard deviation of the robots' speed over ten optimizations with different initial conditions. The results illustrate three interesting properties of this framework. (i) The capability of combining different modes in the fully hybrid setting results in finding solutions with higher values for both average speed and variance in all robot structures, thus resulting in faster and more diverse solutions in the same number of simulations. (ii) Results from pure rotation show a drastic reduction in the robot performance. In the worst case, no viable gait (i.e. one without self-collision) could be found for the Quad5 and Arbit robots. Allowing the robot to lock some of its degrees of freedom, when the others are in rotational mode, helps to avoid self-collision during robot locomotion. The performance of this mode is comparable in terms of characteristics with the oscillation mode since both include locked joints (defined implicitly in oscillation mode due to zero amplitudes). (iii) The results indicate that the performance of both oscillation and hybrid rotation mode are strongly dependent on the robot shape. In 

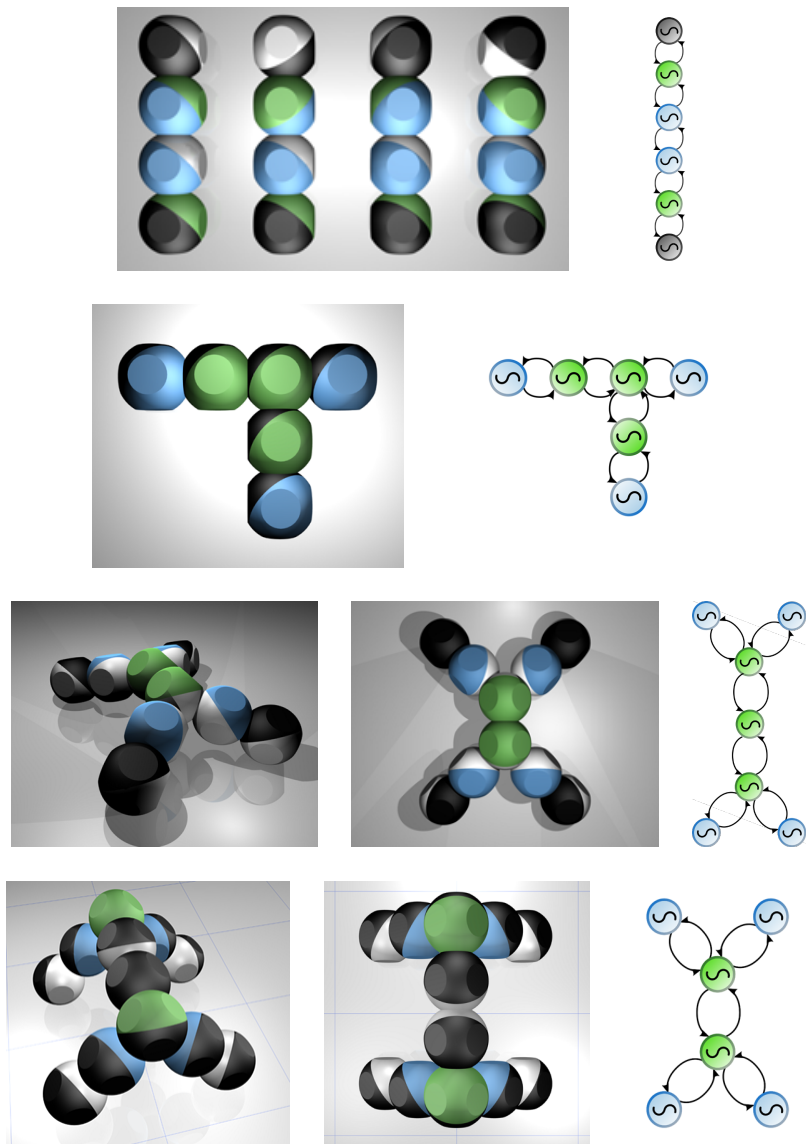

Fig. 4: Pictures of different robot shapes used for our experiments, and their CPG networks: four metamodule configurations; one arbitrary, asymmetric shape with three RB units (Arbit); quadruped with five units (Quad5); quadruped with six units (Quad6). The network mimics the physical topology by coupling neighbor oscillators.

the case of PAR and Quad6, oscillation largely outperforms hybrid rotation. For PER, SRS, SRZ and Quad5 however, similar performance for both modes is observed. Hybrid rotation is only prefered for the Arbit robot. This shows that for a given robot shape, it is not trivial to select either oscillation or hybrid rotation. The complex interaction of the different DOF of a robot shape and the environment determine whether hybrid rotation or oscillation will provide the best performance. In almost all experiments having a mixture of both movement types (fully hybrid) yields better results. Thus, to answer the question of rotation versus oscillation, combining both modes provides the best strategy.

b) Gait diversity: Table II shows the comparison between the different movement type experiments for gait generation of the Quad6 robot. We consider four aspects; (i) the best speed driven by each experiment (ii) the mean value of the speed of the resulting gaits, (iii) the standard deviation of the speed and (iv) the number of different combinations of movement types which are used to generate the gaits. Results in table II show that fully hybrid solutions not only result in average higher speeds, but also allow the robot to derive these solutions by using combinations of joint movements (i.e. have a high "diversity"). Most of the combinations of movement types are hard to imagine and design by hand, because the locomotion behavior results from the complex interactions of the movements of all the degrees of freedom ${ }^{4}$. One can imagine a situation where the algorithm provides an initial selection of possible movement type configurations. A human supervisor can then manually select a few of these configurations, which are of some particular interest, and further optimize the controller for these configurations.

TABLE II: Gait generation results from the different experiments for Quad6

\begin{tabular}{lcccc}
\hline Mode & Best $(\mathrm{m} / \mathrm{s})$ & Mean $(\mathrm{m} / \mathrm{s})$ & Std \% & \# of config. \\
\hline \hline Pure Rotation & 0.22 & 0.17 & 3.1 & 1 \\
Hybrid Rotation & 0.24 & 0.21 & 1.4 & 6 \\
Pure Oscillation & 0.29 & 0.26 & 3.0 & 1 \\
Fully Hybrid & 0.33 & 0.27 & 3.9 & 10 \\
\hline
\end{tabular}

c) Framework performance: Fig. 5 shows the fitness optimization for the best fully hybrid solution of the Quad6 robot. As discussed in section IV, the fully hybrid framework is capable of visiting different combinations of movement types, or sub-swarms, and select the best solution among them. These graphs are related to the sub-swarm with the best speed. Fig. 5a shows the number of fitness evaluations versus the number of iterations. One can see in which iterations and how many times this sub-swarm has been explored. As a result of the mutation probabilities (Fig. 5b), within the first 75 iterations, particles are divided into different subswarms exploring different possible solutions. After iteration 75, exploration stops and the exploitation phase starts where the particles start to be attracted to the best subswarm found in exploration phase. In other words, in the first phase different solutions are explored to find the best candidate and in the second phase these solution spaces are attracting more particles to optimize the open parameters. The plot also shows that to prevent self-collision, several solutions are being penalized by setting their fitness value to zero. Fig. $5 b$ shows how the best fitness of this movement type configuration is evolving through the iterations of the optimization process.
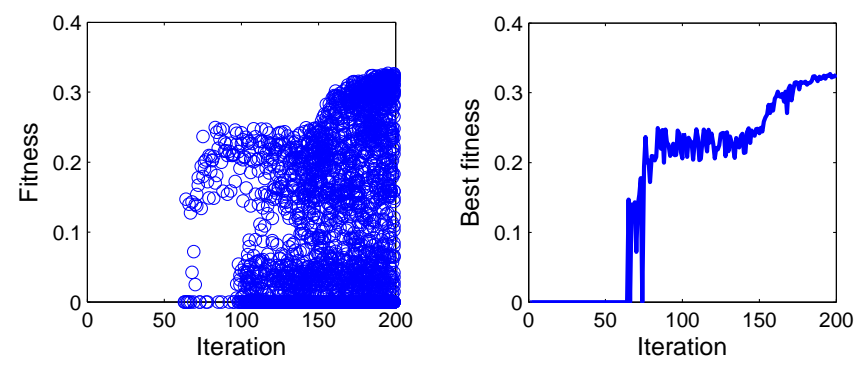

Fig. 5: Fitness optimization of the best fully hybrid solution for Quad5 robot. The figure on the left shows the number of fitness evaluations through the iterations; the figure on the right shows the best fitness for each iteration.

\footnotetext{
${ }^{4}$ Locomotion videos are available at the Roombots webpage: http://biorob.epfl.ch/page38279.html
} 

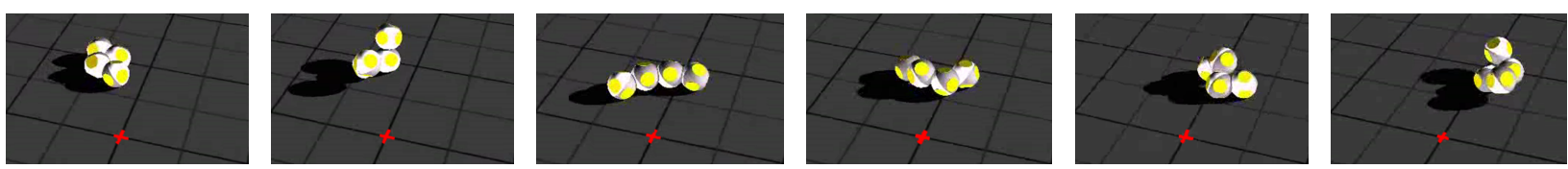

Fig. 7: Snapshots for an evolved gait with a RB PER-metamodule, the module "rolls" from the left to the right. It starts in a folded posture and rotates while unfolding towards the right. While folding top and bottom RB module are switched. This speeds the robot up to $13 \mathrm{~cm} / \mathrm{s}$.
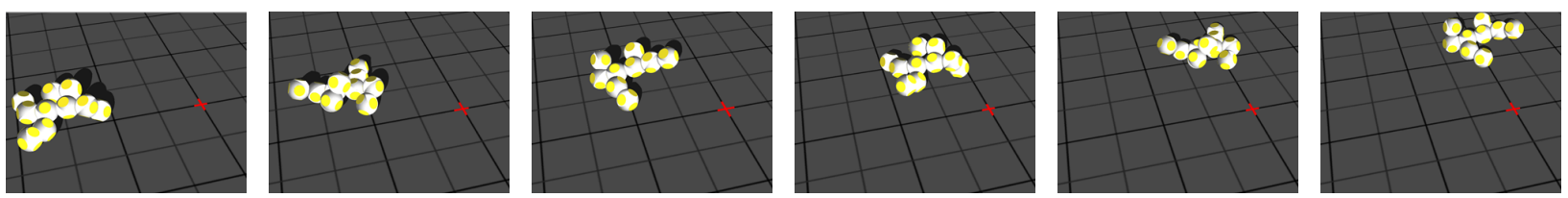

Fig. 8: Fully hybrid optimization on the Quad5 robot, it walks from left to right with $15 \mathrm{~cm} / \mathrm{s}$. Hip joints use rotating patterns, spine joints are blocked. Diagonal limbs are in-phase, and neighboring hind or front limbs are in anti-phase. This results in a trot-like gait.
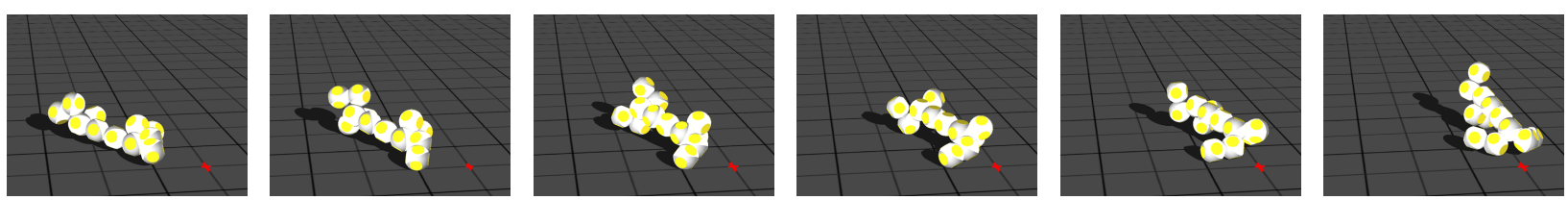

Fig. 9: The Quad6 robot moves from left to the right, half a cycle is shown. It shows one of the fastest "gaits", however does not behave like a typical quadruped. It propels with a winding-like mechanism: by leaving two extremities on the ground it winds the remaining two of them around the body stem and vice versa. Overall direction of movement is sideways with about $29 \mathrm{~cm} / \mathrm{s}$.

d) Gait pattern description: Fig. 7, 8 and 9 show three examples of gait patterns, namely for a PER-metamodule and the two quadruped robots (Fig. 4). Joint movements of the PER-metamodule in Fig. 7 are purely oscillatory, but the robot rolls over itself from cycle to cycle. This is possible in a metamodule-robot because six joints are connected serially-amplitude and velocity are adding up in those structures. The gait (Fig. 9) for the Quad6 robot (Fig. 4) was derived in the fully hybrid optimization mode, and achieves oscillatory and rotational joint movements: both spine joints are in oscillatory mode, one of the outer joints is blocked, one is in rotational mode, and the remaining two leg joints oscillate. The wind-up like gait propels the robot with $29 \mathrm{~cm} / \mathrm{s}$. Average speed for all the quadruped robot gaits derived in fully hybrid mode are approximately $26 \mathrm{~cm} / \mathrm{s}$ (Fig. 6).

e) Advantages from CPG Model: The CPG model has several interesting features that make it well suited for modular robotics. (i) Our model can produce stable rhythmic and rotational patterns such that the dynamical system rapidly returns to its steady state after perturbations of the state variables. (ii) The applied CPG model only needs a few, high level control parameters (in our case amplitude, offset and phase lag). Hence it can reduce the dimensionality of the control problem such that the optimization algorithm only needs to optimize a small number of control signals. (iii) Another useful CPG property is its ability to generate different gaits, which can be achieved by setting the network coupling type and topology. In this way we can reproduce animal-like gaits. This has been done in previous work for quadruped, and modular robots ([6], [24], [25]). Yet one has

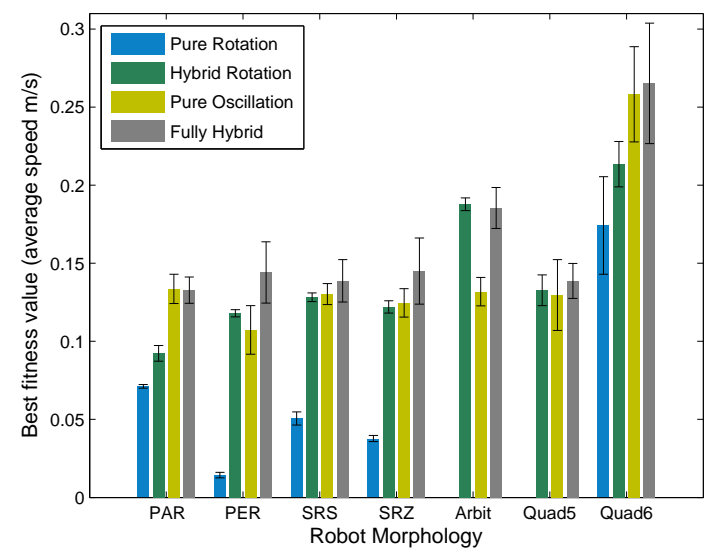

Fig. 6: The optimization results from the seven different robot structures: PAR, PER, SRS and SRZ metamodules, one assymetric robot with 3 modules (arbit) and the two quadruped shapes. Each experiment was repeated ten times, with different initial conditions.

the option to keep the network topology open, and to let new and unexpected gaits emerge. This approach is even more appealing for modular robots, where ideal gaits are initially unknown due to new robot topologies. The proposed framework can reproduce animal-like gaits or find alternative solutions in an automated manner. (iv) This CPG model can be used to generate different types of locomotion patterns. Our control architecture offers a high variety of basic locomotion patterns e.g. it can generate any combination of oscillatory and rotational movements. This allows us to apply those movements to the robot while ensuring that they are in their phase-locked regimes. We observed interesting and 
unexpected locomotion gaits being derived by our combined architecture. In this work we used specific patterns, such as sine-waves for oscillation, and constant speed for rotation. However the framework is kept open and more complex patterns can be implemented, which could lead to an even higher versatility of derived gaits.

\section{CONCLUSION AND FUtURE WORK}

In this paper we have derived a framework for locomotion control of modular robots where a central pattern generator (CPG) as the motion controller and the optimization algorithm are tightly connected. This framework provides an important feature: the optimization algorithm can choose and switch between oscillatory and rotational joint movements, for any joint, at any time during the optimization process, and is fully automated. The user is not required to, but can preassign a movement-type to a joint type. This enables us to derive gait patterns for traditional robots like quadrupeds (oscillatory and stop joint control) but also for robots featuring the more capable, continuous rotational joints, e.g. Whegslike robots or in our case the Roombots modules. We have presented results of our optimization framework deriving pure oscillatory or rotational joint controllers based on CPGs, as well as hybrid controllers. Optimized robot gaits for the latter type often result in mixed-mode joint controllers with surprising characteristics and very competitive performance. In other words it is better to let the optimization algorithm find suitable modes for each joint rather than designing them by hand. Our research on locomotion control will be further pursued in order to address the problems of (i) how to properly include sensory feedback for improving the efficiency and robustness of locomotion patterns, and (ii) of navigation, i.e. how to modulate speed and direction to reach a specific location in a room. We plan to extend the hardware by passive, light-weight elements like carbon-fiber plates to built more complex, and a larger variety of locomotion gaits.

\section{ACKNOWLEDGEMENT}

This project has received funding from the EPFL and from the European Community's Seventh Framework Programme FP7/2007-2013 - Future Emerging Technologies, Embodied Intelligence, under the grant agreements no. 231688 (Locomorph) and no. 231451 (EVRYON). We gratefully acknowledge the technical support of André Guignard, André Badertscher, Peter Brühlmeier, Philippe Voessler, and Manuel Leitos in the design and construction of the robot modules.

\section{REFERENCES}

[1] R. Playter, M. Buehler, and M. Raibert, "BigDog," in Proceedings of SPIE, vol. 6230, 2006, p. 623020.

[2] U. Saranli, M. Buehler, and D. Koditschek, "Rhex: A simple and highly mobile hexapod robot," The International Journal of Robotics Research, vol. 20, no. 7, p. 616, 2001.

[3] A. Ijspeert, A. Crespi, D. Ryczko, and J. Cabelguen, "From swimming to walking with a salamander robot driven by a spinal cord model," Science, vol. 315, no. 5817, p. 1416, 2007.
[4] V. Zykov, J. Bongard, and H. Lipson, "Evolving dynamic gaits on a physical robot," in Proceedings of Genetic and Evolutionary Computation Conference, Late Breaking Paper, GECCO, vol. 4. Citeseer, 2004.

[5] M. Hancher and G. Hornby, "Evolving Quadruped Gaits with a Heterogeneous Modular Robotic System,” pp. 3631-3638, 2007.

[6] A. Sproewitz, R. Moeckel, J. Maye, and A. J. Ijspeert, "Learning to move in modular robots using central pattern generators and online optimization," The International Journal of Robotics Research, vol. 27, no. 3-4, pp. 423-443, mar 2008.

[7] D. Christensen, M. Bordignon, U. Schultz, D. Shaikh, and K. Stoy, "Morphology independent learning in modular robots," in Proc., International Symposium on Distributed Autonomous Robotic Systems (DARS). Springer, 2008.

[8] R. T. Schroer, M. J. Boggess, R. J. Bachmann, R. D. Quinn, and R. E. Ritzmann, "Comparing cockroach and whegs robot body motion," Proceedings of the IEEE International Conference on Robotics and Automation 2004, pp. 3288-3293, apr 2004.

[9] V. Zykov, P. William, N. Lassabe, and H. Lipson, "Molecubes Extended: Diversifying Capabilities of Open-Source Modular Robotics," in IROS-2008 Self-Reconfigurable Robotics Workshop, accepted, 2008.

[10] M. Yim, "Locomotion with a unit modular reconfigurable robot," $\mathrm{Ph} . \mathrm{D}$. dissertation, Stanford University Mechanical Engineering Dept., 1994.

[11] Y. Zhang, M. Yim, C. Eldershaw, D. Duff, and K. Roufas, "Scalable and reconfigurable configurations and locomotion gaits for chaintype modular reconfigurable robots," in International Symposium on Computational Intelligence in Robotics and Automation, 2004, pp. 893-899.

[12] J. Bongard, V. Zykov, and H. Lipson, "Resilient machines through continuous self-modeling," Science, vol. 314 , no. 5802, pp. 1118 1121, 2006.

[13] K. Stoy, W. Shen, and P. Will, "Implementing configuration dependent gaits in a self-reconfigurable robot," in Proceedings of ICRA2003, 2003.

[14] W. Shen, P. Will, A. Galstyan, and C. Chuong, "Hormone-inspired self-organization and distributed control of robotic swarms," $A u$ tonomous Robots, vol. 17, no. 1, pp. 93-105, 2004.

[15] Y. Zhang, M. Fromherz, L. Crawford, and Y. Shang, "A general constraint-based control framework with examples in modular selfreconfigurable robots," in IEEE/RSJ International Conference on Intelligent Robots and Systems (IROS2002), 2002.

[16] A. Kamimura, H. Kurokawa, E. Toshida, K. Tomita, S. Murata, and S. Kokaji, "Automatic locomotion pattern generation for modular robots," in IEEE International Conference on Robotics and Automation (ICRA2003), 2003.

[17] K. Sims, "Evolving 3d morphology and behavior by competition," in Proceedings, Artificial Life IV. MIT Press, 1994, pp. 28-39.

[18] A. Sproewitz, A. Billard, P. Dillenbourg, and A. J. Ijspeert, "Roombots-mechanical design of self-reconfiguring modular robots for adaptive furniture," in 2009 IEEE International Conference on Robotics and Automation, Kobe, Japan, 2009, pp. 4259-4264.

[19] A. Sproewitz, M. Asadpour, Y. Bourquin, and A. Ijspeert, "An active connection mechanism for modular self-reconfigurable robotic systems based on physical latching," in Robotics and Automation, 2008. ICRA 2008. IEEE International Conference on, 2008, pp. 3508-3513.

[20] Y. Terada and S. Murata, "Automatic modular assembly system and its distributed control," The International Journal of Robotics Research, vol. 27, no. 3-4, pp. 445-462, mar 2008.

[21] J. Kennedy and R. Eberhart, "Particle swarm optimization," in "Proceedings of IEEE International Conference on Neural Networks", vol. 4, 1995, pp. 1942-1948.

[22] M. Clerc and J. Kennedy, "The particle swarm - explosion, stability, and convergence in a multidimensional complex space," "Evolutionary Computation, IEEE Transactions on", vol. 6, no. 1, pp. 58-73, 2002.

[23] Webots, "http://www.cyberbotics.com," commercial Mobile Robot Simulation Software. [Online]. Available: http://www.cyberbotics.com

[24] H. Kimura, Y. Fukuoka, and A. H. Cohen, "Adaptive dynamic walking of a quadruped robot on natural ground based on biological concepts," The International Journal of Robotics Research, vol. 26, no. 5, pp. 475-490, may 2007.

[25] A. Kamimura, H. Kurokawa, E. Yoshida, S. Murata, K. Tomita, and S. Kokaji, "Distributed adaptive locomotion by a modular robotic system, M-TRAN II," in Proceedings of the IEEE/RSJ IROS2004, 2004, pp. 2370-2377. 